\title{
Evaluation of Various Drugs' Influence on Alzheimer's Disease Progress Using a New Analytical Model Based on Cellular Automata
}

\author{
Niloofar Jafari ${ }^{1}$, Faegheh Golabi ${ }^{1 *}$, Abbas Ebrahimi-kalan², Yashar Sarbaz ${ }^{3 *}$ \\ ${ }^{1}$ Department of Biomedical Engineering, Faculty of Advanced Medical Sciences, Tabriz University of Medical \\ Sciences, Tabriz, Iran \\ ${ }^{2}$ Department of Neuroscience and Cognition, Faculty of Advanced Medical Sciences, Tabriz University of Medical \\ Sciences, Tabriz, Iran \\ ${ }^{3}$ Department of Biomedical Engineering, Faculty of Electrical and Computer Engineering, University of Tabriz, \\ Tabriz, Iran
}

${ }^{*}$ Corresponding authors:

Faegheh Golabi,

Department of Biomedical Engineering,

Faculty of Advanced Medical Sciences,

Tabriz University of Medical Sciences,

Tabriz, Iran

faegheh.golabi@gmail.com.

Yashar Sarbaz,

Department of Biomedical Engineering,

Faculty of Electrical and Computer Engineering,

University of Tabriz,

Tabriz, Iran

yashar.sarbaz@tabrizu.ac.ir.

\begin{abstract}
This article aims to introduce and propose a novel mathematical model for the study of Alzheimer's disease (AD) progress. The presented model is based on Cellular Automata for better representation of $\mathrm{AD}$ progression. The differential equations of the Puri-Li model are utilized to calculate the number of Amyloid- $\beta$ molecules. Also, a new definition for AD rate is presented in this study. Moreover, other useful factors such as Critical Rate (CR) and Warning Rate (WR) are defined to determine the status of $\mathrm{AD}$ progression. To get exact insight into the neuron-to-neuron communications, the model is obtained for a $3 \times 3$ neuron system to investigate the influence of drug injection on the reduction of AR, CR, and WR factors. It is shown that using drugs can decrease AR and CR factors and also enhance the WR. The presented study can be utilized for the investigation of various factors in the control and treatment of $\mathrm{AD}$ progression.
\end{abstract}

Keywords: Alzheimer's disease, Cellular Automata, Analytical Model, Drug, Amyloid- $\beta$ 


\section{Introduction}

Mathematical modeling is a powerful method for the forecasting, treatment, and study of various brain disorders $[1,2]$. This method assists the biologists in exactly following the progress of the specific disease and also investigating the effect of various external factors such as drugs, music, exercise, and gamma-ray on the progression of brain disorders. One of the famous neurodegenerative disorders that have affected millions of people all over the world is Alzheimer's disease $(A D)$, which is mainly caused by the agglomeration of Amyloid- $\beta$ (A $\beta$ ) molecules within the hippocampus [3].

Analytical modeling of AD has been published in many research works [4-19]. One of the famous models in this research area is the Puri-Li model which suggests seven differential rate equations to consider the cross-talk between neuron, astroglia, microglia, and A $\beta$. Many kinds of research are conducted based on this model, for instance, the effect of calcium Ion Hemostasis on AD progress is investigated, based on the Puri-Li model [7].

In this article, by merging the differential equation of the Puri-Li model and Cellular Automata (CA), we introduce novel analytical mathematical relations to model the neuron-to-neuron communications in a $3 \times 3$ neuron system. As we know, CA is one of the powerful methods which assists biologists to schematically observe and follow the progression of brain disorders. We also investigate the drugs' effect on the reduction of AD progress, which has a large impact on AD. It is shown that the use of suitable drugs can decrease AR and CR factors and also enhance the WR.

\section{Analytical Model}

Fig. 1 represents the biological structure of the neurons. As seen in Fig. 1 (a), each neuron has three main parts: cell body, axons, and dendrites. To model the neuron, as shown in Fig. 1 (b), we have ignored axon, Myelin sheath, and node of Ranvier and only kept the cell body of the neuron together with its synapses. As we know, the agglomeration of A $\beta$ molecules occurs on synapses continuously by forming platelets. Therefore, considering the cell body of the neuron with its synapses is a realistic supposition in this study. Furthermore, the synapses have been nominated in a clockwise direction to show the formation of continuous platelets on synapses, as seen in Fig. 1 (c). We assume that each neuron has about synapse total $=1000$ synapses, which means that each square synapse cell (i.e. $\left.S_{1}, S_{2}, S_{3}, S_{4}, S_{5}, S_{6}, S_{7}, S_{8}\right)$ contains synapse $_{\text {per cell }}=$ synapse $_{\text {total }} / 8=125$ synapses. 


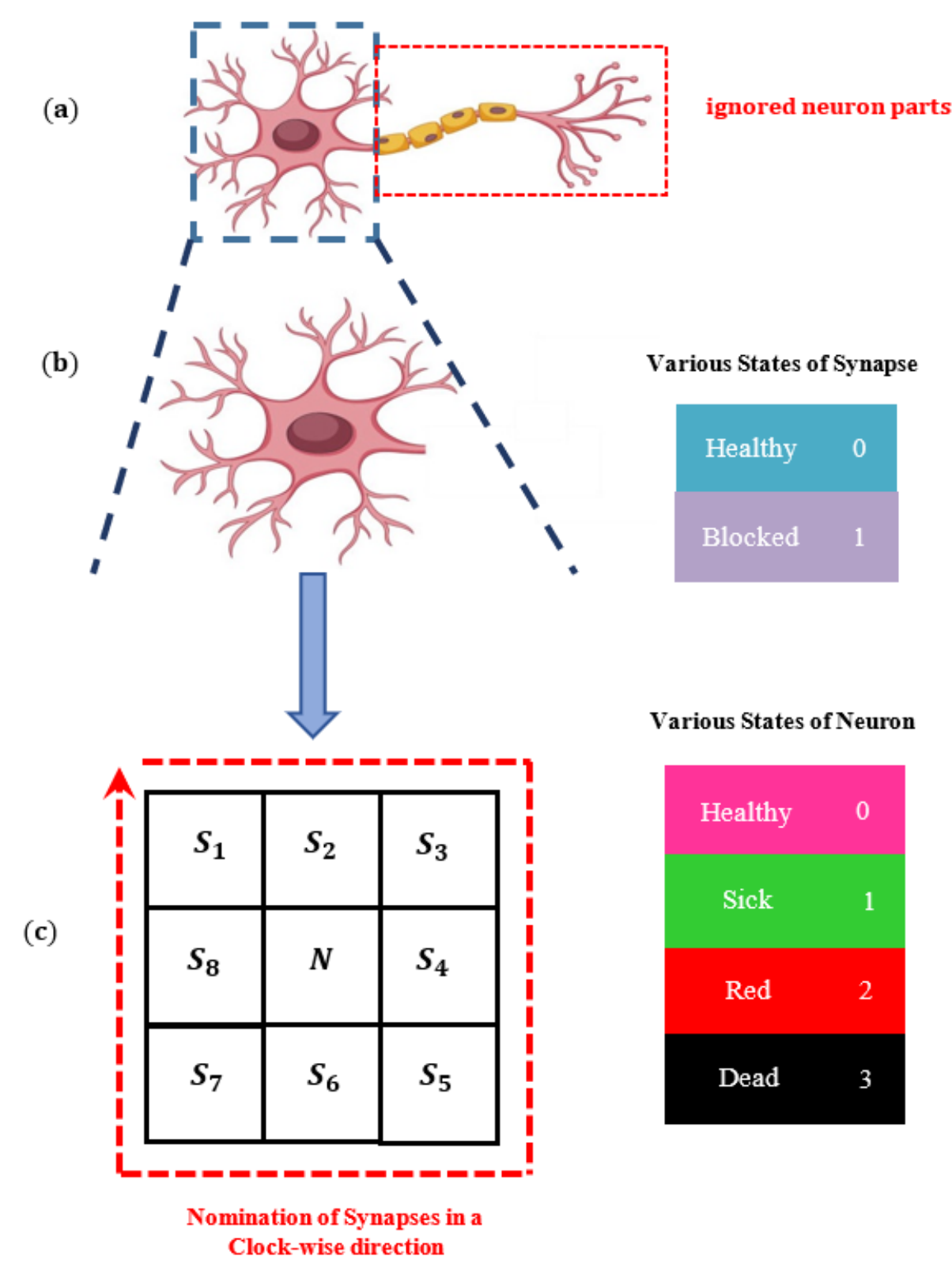

Fig. 1. (a) Various parts of a neuron, including cell body, an axon, and dendrites, (b) The cell body (core) and its adjacent synapses, (c) CA Network constructed of a central cell for neuron (core) and eight cells for all its synapses. The synapse cells are named/numbered in a clockwise direction.

In our model, each synapse cell has two possible states while the central cell has 4 states. State " 0 " (Healthy) for a synapse cell implies a situation where the amount of $A \beta$ molecules is not sufficient to block all synapses of the synapse cell, while state "1" (Blocked) is utilized for a synapse cell in which its whole synapses are blocked by the sufficient value of $\mathrm{A} \beta$ molecules. Meanwhile as illustrated in Fig. 1 (c), the neuron itself can be in one of four states including state " 0 " (Healthy), state "1" (sick), state " 2 " (red), and state " 3 " (dead). The healthy state implies a 
neuron in which its connections with other neurons are retained due to the low value of $A \beta$ molecules and consequently low number of blocked synapse cells. For a dead neuron (state " 3 "), neuron-to-neuron connections are interrupted because more than $50 \%$ of the synapses have been blocked by the agglomeration of A $\beta$ molecules. Usually, only two states (i.e., alive or dead neurons) are considered for a neuron in the literature. Since the healthy neuron gradually converts into a dead neuron, we define two new states for a neuron, i.e., sick and red states. The state " 1 " (sick state) is a neuron in which $25 \%$ of its synapse are blocked while the state " 2 " (red state) is an emergency state of a neuron in which between $25 \%$ up to $50 \%$ of its synapses are blocked.

Let us suppose that the hippocampus, a small area within the brain that the AD begins, occupies about $2.5 \mathrm{~cm}^{2}$ in the two-dimensional surface. The number of cells is about $10^{6}$ by assuming that the effective area of each neuron is $250 \mu \mathrm{m}^{2}$. This article aims to get biological insight to determine how the progression of $\mathrm{AD}$ occurs and the simulation of these huge numbers is outside the scope of this article. Therefore, we consider a $3 \times 3$ neuron network here, as depicted in Fig. 2.

\begin{tabular}{|c|c|c|c|c|c|c|c|c|}
\hline$S_{1,1}$ & $S_{1,2}$ & $S_{1,3}$ & $S_{2,1}$ & $S_{2,2}$ & $S_{2,3}$ & $S_{3,1}$ & $S_{3,2}$ & $S_{3,3}$ \\
\hline$S_{1,8}$ & $N_{1}$ & $S_{1,4}$ & $S_{2,8}$ & $N_{2}$ & $S_{2,4}$ & $S_{3,8}$ & $N_{3}$ & $S_{3,4}$ \\
\hline$S_{1,7}$ & $S_{1,6}$ & $S_{1,5}$ & $S_{2,7}$ & $S_{2,6}$ & $S_{2,5}$ & $S_{3,7}$ & $S_{3,6}$ & $S_{3,5}$ \\
\hline$S_{4,1}$ & $S_{4,2}$ & $S_{4,3}$ & $S_{5,1}$ & $S_{5,2}$ & $S_{5,3}$ & $S_{6,1}$ & $S_{6,2}$ & $S_{6,3}$ \\
\hline$S_{4,8}$ & $N_{4}$ & $S_{4,4}$ & $S_{5,8}$ & $N_{5}$ & $S_{5,4}$ & $S_{6,8}$ & $N_{6}$ & $S_{6,4}$ \\
\hline$S_{4,7}$ & $S_{4,6}$ & $S_{4,5}$ & $S_{5,7}$ & $S_{5,6}$ & $S_{5,5}$ & $S_{6,7}$ & $S_{6,6}$ & $S_{6,5}$ \\
\hline$S_{7,1}$ & $S_{7,2}$ & $S_{7,3}$ & $S_{8,1}$ & $S_{8,2}$ & $S_{8,3}$ & $S_{9,1}$ & $S_{9,2}$ & $S_{9,3}$ \\
\hline$S_{7,8}$ & $N_{7}$ & $S_{7,4}$ & $S_{8,8}$ & $N_{8}$ & $S_{8,4}$ & $S_{9,8}$ & $N_{9}$ & $S_{9,4}$ \\
\hline$S_{7,7}$ & $S_{7,6}$ & $S_{7,5}$ & $S_{8,7}$ & $S_{8,6}$ & $S_{8,5}$ & $S_{9,7}$ & $S_{9,6}$ & $S_{9,5}$ \\
\hline
\end{tabular}

Fig. 2. Cellular automata for $3 \times 3-$ neuron network.

As explained before, the main parameter in $\mathrm{AD}$ is the amount of $\mathrm{A} \beta$ molecules, which can be calculated by the Puri-Li dynamic model for each neuron in a $3 \times 3$ network [4]: 


$$
\begin{array}{cc}
\frac{d X_{i}}{d t}=A_{i} X_{i} & i=1,2,3, \ldots, 9 \\
X_{i}=\left[\begin{array}{c}
N_{\text {Healthy|Sick }} \\
N_{\text {Red|Dead }} \\
A_{q} \\
A_{p} \\
M_{2} \\
M_{1} \\
A \beta
\end{array}\right]_{i} \quad i=1,2,3, \ldots, 9
\end{array}
$$

where

$$
A=\left[\begin{array}{ccccccc}
0 & 0 & \alpha_{1} & -\alpha_{2} & 0 & -\alpha_{3} & 0 \\
0 & 0 & -\alpha_{1} & +\alpha_{2} & 0 & +\alpha_{3} & 0 \\
0 & 0 & 0 & 0 & \alpha_{4} & -\alpha_{5} & 0 \\
0 & 0 & 0 & 0 & -\alpha_{4} & \alpha_{5} & 0 \\
\left(\alpha_{6}+\alpha_{11}\right) & -\alpha_{10} & \left(\alpha_{7}+\alpha_{12}\right) & 0 & \alpha_{14} & -\alpha_{9} & -\left(\alpha_{8}+\alpha_{13}\right) \\
-\left(\alpha_{6}+\alpha_{11}\right) & \alpha_{10} & -\left(\alpha_{7}+\alpha_{12}\right) & 0 & -\alpha_{14} & \alpha_{9} & \left(\alpha_{8}+\alpha_{13}\right) \\
\alpha_{15} & 0 & 0 & 0 & -\alpha_{16} & 0 & 0
\end{array}\right]
$$

is a matrix, as defined by Puri and Li [4]. In (2), $A_{q}, A_{p}, M_{2}, M_{1}, A \beta$ are the number of quiescent astroglia, proliferating astroglia, anti-inflammatory state, pro-inflammatory state, and $\mathrm{A} \beta$ molecules, respectively [4]. In the general form, one can express the response of (1-3) for $i$-th neuron (the primary values of $x_{0, i}$ ) as follows:

$$
\begin{aligned}
& X_{i}(\mathrm{t})=\exp (A t) \cdot x_{0, i} ; \exp (A t)=L^{-1}\left[(s I-A)^{-1}\right] \\
& x_{0, i}=\left[\begin{array}{c}
N_{\text {Healthy|Sick }} \\
N_{\text {Red } \mid \text { Dead }} \\
A_{q} \\
A_{p} \\
M_{2} \\
M_{1} \\
A \beta
\end{array}\right]_{0, i} \quad i=1,2,3, \ldots, 9
\end{aligned}
$$

In equation (4), $A \beta$ determines the whole value of $A \beta$ molecules for all synapses of the $i$-th neuron. Now, the number of blocked synapse cells at $t=t_{0}$ is obtained: 
Synapses $_{\text {Blocked }, i}=\left[\frac{A \beta_{\text {total }, i}\left(\mathrm{t}=\mathrm{t}_{0}\right)}{\frac{A \beta_{\max }}{\text { Synapse }_{\text {total }}} \times \text { Synapse }_{\text {per cell }}}\right]=\left[\frac{A \beta_{\text {total }, i}\left(\mathrm{t}=\mathrm{t}_{0}\right)}{\frac{A \beta_{\max }}{\text { Synapse }_{\text {total }}} \times \frac{\text { Synapse }_{\text {total }}}{8}}\right]=\left[\frac{A \beta_{\text {total }, i}\left(t=t_{0}\right)}{\frac{A \beta_{\max }}{8}}\right]$

$i=1,2,3, \ldots, 9$

Based on equation (5), $A \beta_{\text {total,N }}\left(t=t_{0}\right)$ and $A \beta_{\max }$ are the value of $\mathrm{A} \beta$ molecules (calculated by the Puri-Li model) and the maximum value of $\mathrm{A} \beta$ molecules to be blocked completely, respectively. Moreover, the "[]" is used for the floor function. Now, the synapse states for the $i$-th neuron can be derived:

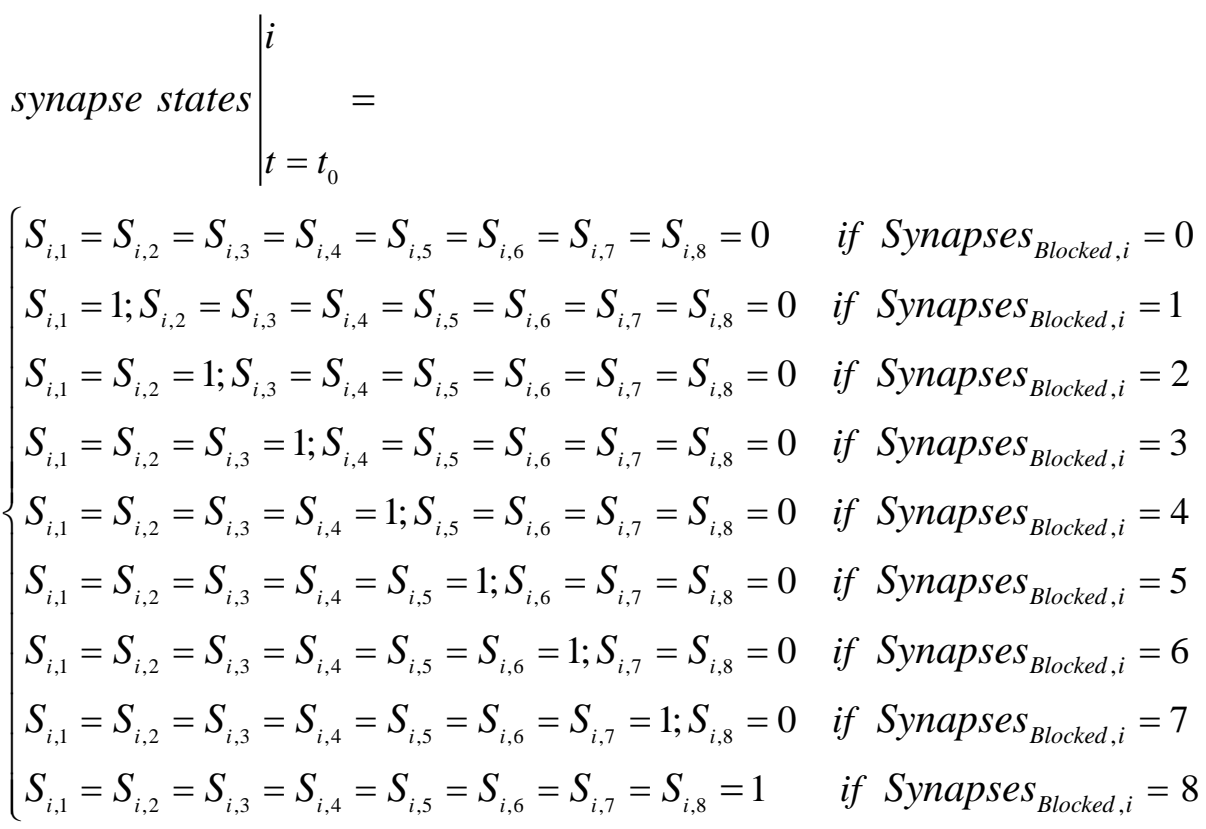

It should be explained that the presented formula in (5) cannot model the neuron-to-neuron communications effectively since it only calculates the number of self-blocked synapse cells. Within the brain, the synapses of each neuron send/receive signals to/from adjacent and nonadjacent neurons. Here, neuron-to-neuron communication of adjacent neurons is modeled for a $3 \times 3$ - neuron network:

$$
\begin{aligned}
& \text { Synapses }_{\text {Blocked }, i}^{\text {total }}=\text { Synapses }_{\text {Blocked }, i}^{\text {Self }}+\text { Synapses }_{\text {Blocked }, i}^{\text {Adjaront Neurs }}= \\
& {\left[\frac{A \beta_{\text {total }, i}\left(t=t_{0}\right)}{\frac{A \beta_{\text {total,max }}}{8}}\right]+\text { Synapses }_{\text {Blocked }, i}^{\text {Adjacent }} \quad i=1,2,3, \ldots, 9}
\end{aligned}
$$


As shown in Fig. 3, in our model of $3 \times 3-$ Neuron Network, three kinds of neurons exist: 1 Corner Neurons (i.e. $N_{1}, N_{3}, N_{7}, N_{9}$, their communications are illustrated by green color), 2- Row and Column Neurons (i.e. $N_{2}, N_{4}, N_{6}, N_{8}$, their communications are shown by blue color), and 3Central Neuron (i.e. $N_{5}$, it has been highlighted by brown color). The neuron state for each kind of these categories can be obtained as follows:

$$
\text { Neuron states } \mid \begin{aligned}
& \text { for Corner Neurons }(i=1,3,7,9) \\
& t=t_{0}
\end{aligned}= \begin{cases}0 & \text { if } 0 \leq \text { Synapses }_{\text {Blocked }, i}^{\text {total }} \leq 2 \\
1 & \text { if } 3 \leq \text { Synapses }_{\text {Blocked }, i}^{\text {total }} \leq 4 \\
2 & \text { if } 5 \leq \text { Synapses }_{\text {Blocked }, i}^{\text {total }}<8 \\
3 & \text { if Synapses } \text { Blocked }, i^{\text {total }} \geq 8\end{cases}
$$

Neuron states $\mid \begin{aligned} & \text { for Row and Column Neurons }(i=2,4,6,8) \\ & t=t_{0}\end{aligned}=$

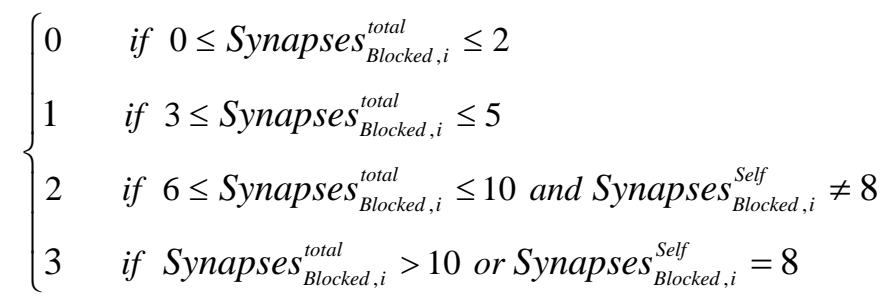

Neuron states $\mid \begin{aligned} & \text { for Central Neuron }(i=5) \\ & t=t_{0}\end{aligned}= \begin{cases}0 & \text { if } 0 \leq \text { Synapses }_{\text {Blocked }, i}^{\text {total }} \leq 3 \\ 1 & \text { if } 4 \leq \text { Synapses }_{\text {Blocked }, i}^{\text {total }} \leq 6 \\ 2 \quad & \text { if } 7 \leq \text { Synapses }_{\text {Blocked }, i}^{\text {total }} \leq 12 \text { and } \text { Synapses }_{\text {Blocked }, i}^{\text {Self }} \\ 3 \quad & \text { if } \text { Synapses }_{\text {Blocked }, i}^{\text {total }}>12 \text { or Synapses } \\ \text { Blocked }, i\end{cases}$

To effectively control the individual status, the following parameters are defined:

Alzheimer's Rate $(A R)=\frac{\text { Number of Dead Neurons }}{M \times L}$

Critical Rate $(C R)=\frac{\text { Number of Red Neurons }}{M \times L}$

Warning Rate $($ WR $)=\frac{\text { Number of Sick Neurons }}{M \times L}$ 


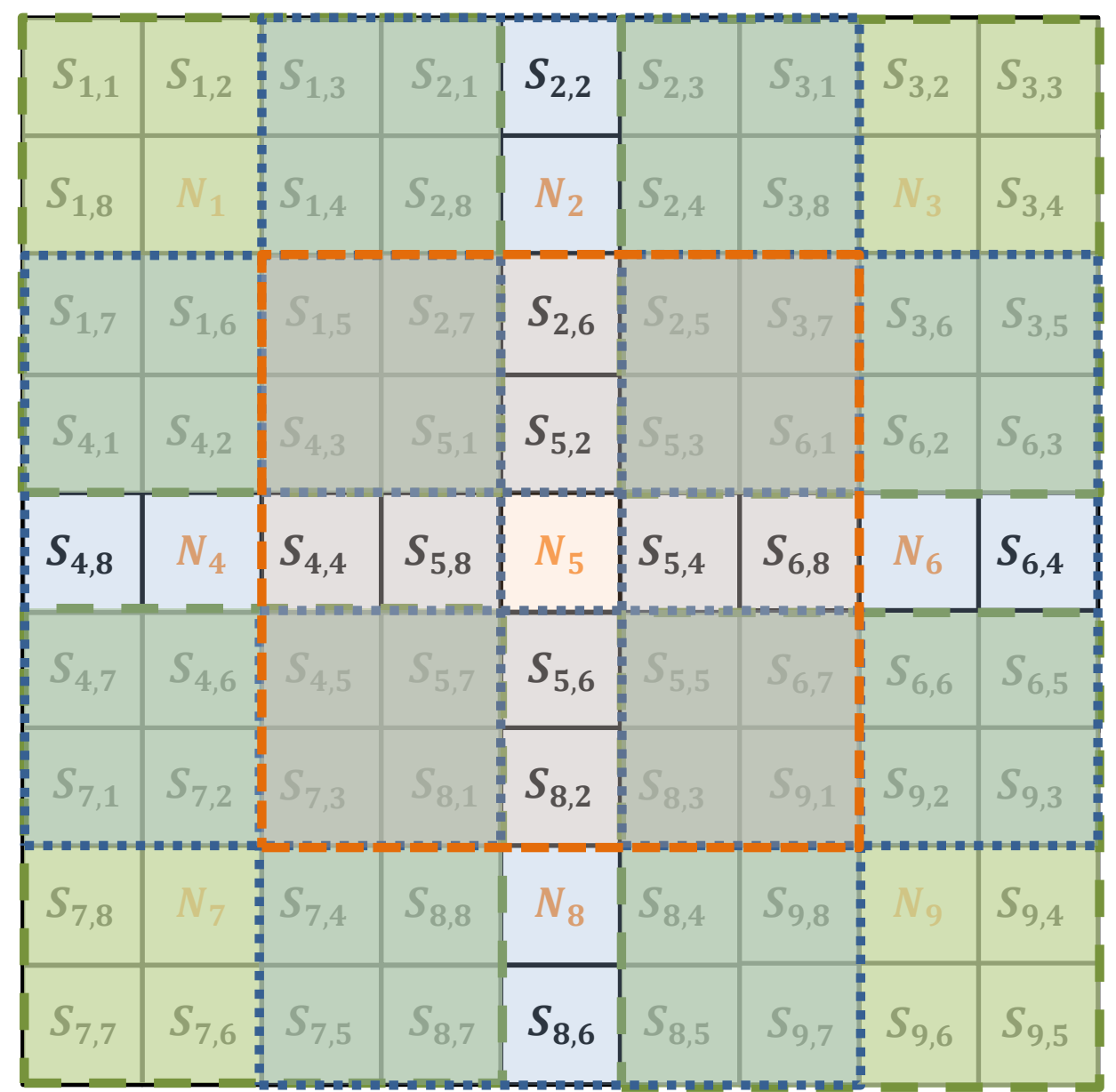

Fig. 3. Neuron-to-neuron communications in $3 \times 3-$ neuron network. Corner neurons, row, and column neurons, and central neurons are illustrated by green, blue, and brown colors, respectively.

By supposing the time step of $t=t_{0}$, the synapse state for the next time step, i.e., $t=2 t_{0}$, can be obtained by using Puri-Li equations:

$$
x_{t=t_{0}, i} \Rightarrow A \beta_{\text {total }, i}\left(t=2 t_{0}\right) \Rightarrow \text { synapse states }\left.\right|_{t=2 t_{0}} ^{i} i=1,2,3, \ldots, 9
$$

However, some internal mechanisms within the brain and also some external factors such as drugs, can modify the amount of $\mathrm{A} \beta$ molecules. Therefore, the primary values for $i$-th neuron at $t=t_{0}$ can be updated as: 


$$
x_{t=t_{0}, i}^{\text {modified }}=\left[\begin{array}{c}
N_{\text {Healthy } \mid \text { Sick }, t=t_{0}, i} \\
N_{\text {Red } \mid \text { Dead }, t=t_{0}, i} \\
A_{q, t=t_{0}, i} \\
A_{p, t=t_{0}, i} \\
M_{2, t=t_{0}, i} \\
M_{1, t=t_{0}, i}^{\text {modified }_{t=t_{0}, i}}
\end{array}\right] \quad i=1,2,3, \ldots, 9
$$

where $A \beta_{t=t_{0}, i}^{\text {modified }}$ in (15) is defined as:

$$
\begin{aligned}
& A \beta_{t=t_{0}, i}^{\text {modified }}=A \beta_{t=t_{0}, i} \times\left(1-\left[P_{i}^{\text {Internal Factors }}+P_{i}^{\text {External Factors }}\right]\right) \quad i=1,2,3, \ldots, 9 \\
& P_{i}^{\text {Internal Factors }}= \begin{cases}P_{1 \rightarrow 0}^{\text {Internal Factors }} & \text { if the state of } i-\text { th Neuron is } 1 \\
P_{2 \rightarrow 1}^{\text {Internal Factors }} & \text { if the state of } i-\text { th Neuron is } 2\end{cases} \\
& P_{i}^{\text {External Factors }}= \begin{cases}P_{1 \rightarrow 0}^{\text {External Factors }} & \text { if the state of } i-\text { th Neuron is } 1 \\
P_{2 \rightarrow 1}^{\text {External Factors }} & \text { if the state of } i-\text { th Neuron is } 2\end{cases}
\end{aligned}
$$

For instance, $P_{1 \rightarrow 0}^{\text {Internal Factors }}$ is the probability of the state of the $i$-th neuron changing from the sick state (state " 1 ") to the healthy state (state " 0 ") as result of internal factors.

\section{Results and Discussions}

This section studies the numerical results of the proposed model for a $3 \times 3$ neuron network coded by software. The simulation parameters of the CA model and the mathematical parameters of the Puri-Li model are given in Table. 1 and Table. 2, respectively. In Table. 3, the probabilistic functions for three AD drugs, i.e., Rivastigmine, Donepezil, and Galantamine, are given. Moreover, the initial values of the neuron parameters have been given in Table. 4.

In Fig. 4, the CA representation of neurons and their synapses for a sample $3 \times 3$ neuron network has been depicted at $t=0$ and $t=1$ year. As mentioned previously, the status of each neuron depends on the states of its synapses and the synapses of adjacent neurons. For instance, all synapses of neuron $(1,3)$ are healthy in Fig. 4 (a) while its status is sick, due to the blocked synapse cells of its adjacent neurons (i.e., neuron $(1,2)$ and neuron $(2,3)$ ). After one year, although $\mathrm{AR}$ is still zero, $\mathrm{CR}$ reaches $88.8 \%$, which is a critical situation. To control this critical situation, by reducing the $\mathrm{CR}$ factor, some internal mechanisms within the hippocampus can help to decrease the amount of $\mathrm{A} \beta$. These mechanisms are essential but not adequate. Therefore, an effective external factor should be added to this system to reduce CR. One practical way is the usage of drugs such as Rivastigmine, Donepezil, and Galantamine. Here, we aim to investigate the influence of these drugs on the enhancement of critical situations in a $3 \times 3$ neuron network. 
Table 1. Simulation Parameters of CA Network.

\begin{tabular}{|c|c|c|c|c|c|c|c|}
\hline Parameter & Synapse $_{\text {total }}$ & Synapse $_{\text {per cell }}$ & $\mathrm{A} \beta_{\max }$ & Time step $\left(\mathrm{t}_{0}\right)$ & Network $(\mathrm{M} \times \mathrm{L})$ & $\mathrm{P}_{1 \rightarrow 0}^{\text {Internal }}$ & $\mathrm{P}_{2 \rightarrow 1}^{\text {Internal }}$ \\
\hline Value & 1000 & 125 & 8 & 1 year & $3 \times 3$ & 0.7 & 0.3 \\
\hline
\end{tabular}

Table 2. Mathematical Parameters of Puri-Li model (1/year).

\begin{tabular}{c||c|c|c|c|c|c|c|c|c|c|c|c|c|c|c|c}
\hline \hline Parameter & $\alpha_{1}$ & $\alpha_{2}$ & $\alpha_{3}$ & $\alpha_{4}$ & $\alpha_{5}$ & $\alpha_{6}$ & $\alpha_{7}$ & $\alpha_{8}$ & $\alpha_{9}$ & $\alpha_{10}$ & $\alpha_{11}$ & $\alpha_{12}$ & $\alpha_{13}$ & $\alpha_{14}$ & $\alpha_{15}$ & $\alpha_{16}$ \\
\hline Value & $10^{-5}$ & $10^{-3}$ & $10^{-2}$ & $10^{-4}$ & $10^{-2}$ & $10^{-2}$ & $10^{-4}$ & $10^{-2}$ & $10^{-2}$ & $10^{-2}$ & $10^{-2}$ & $10^{-4}$ & $10^{-2}$ & $10^{-4}$ & 1 & $10^{-2}$ \\
\hline \hline
\end{tabular}

Table 3. Probabilistic functions of various drugs.

\begin{tabular}{c|c||c|c||c|c}
\hline \hline \multicolumn{2}{c||}{ Rivastigmine } & \multicolumn{2}{c||}{ Donepezil } & \multicolumn{2}{c}{ Galantamine } \\
\hline \hline $\mathrm{P}_{1 \rightarrow 0}^{\text {External }}$ & $\mathrm{P}_{2 \rightarrow 1}^{\text {External }}$ & $\mathrm{P}_{1 \rightarrow 0}^{\text {External }}$ & $\mathrm{P}_{2 \rightarrow 1}^{\text {External }}$ & $\mathrm{P}_{1 \rightarrow 0}^{\text {External }}$ & $\mathrm{P}_{2 \rightarrow 1}^{\text {External }}$ \\
\hline 0.08 & 0.07 & 0.19 & 0.17 & 0.16 & 0.15 \\
\hline \hline
\end{tabular}

Table 4. Initial values of various parameters of neurons in a $3 \times 3$ network.

\begin{tabular}{|c|c|c|c|c|c|}
\hline \multirow{2}{*}{ Neuron $(i, j)$} & \multicolumn{5}{|c|}{ Parameters } \\
\hline & $A_{q}$ & $A_{p}$ & $M_{1}$ & $M_{2}$ & $A \beta$ \\
\hline$(1,1)$ & 10 & 0.1 & 0.1 & 11 & 0.2 \\
\hline$(1,2)$ & 32 & 0.52 & 0.5 & 28 & 3.4 \\
\hline$(1,3)$ & 10 & 0.1 & 0.11 & 10 & 0.12 \\
\hline$(2,1)$ & 13 & 0.25 & 0.2 & 12 & 0.8 \\
\hline$(2,2)$ & 14 & 0.23 & 0.22 & 13.2 & 1.1 \\
\hline$(2,3)$ & 19.7 & 0.45 & 0.41 & 14 & 2.18 \\
\hline$(3,1)$ & 43 & 0.57 & 0.51 & 31 & 4.5 \\
\hline$(3,2)$ & 19.6 & 0.44 & 0.38 & 13.8 & 2.17 \\
\hline$(3,3)$ & 64 & 0.59 & 0.61 & 53 & 6.2 \\
\hline
\end{tabular}

(a) at $\mathrm{t}=0$

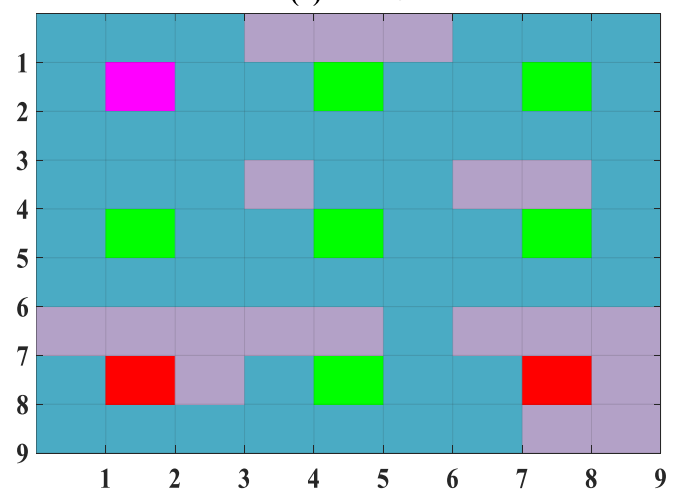

(b) at $\mathrm{t}=1$ year

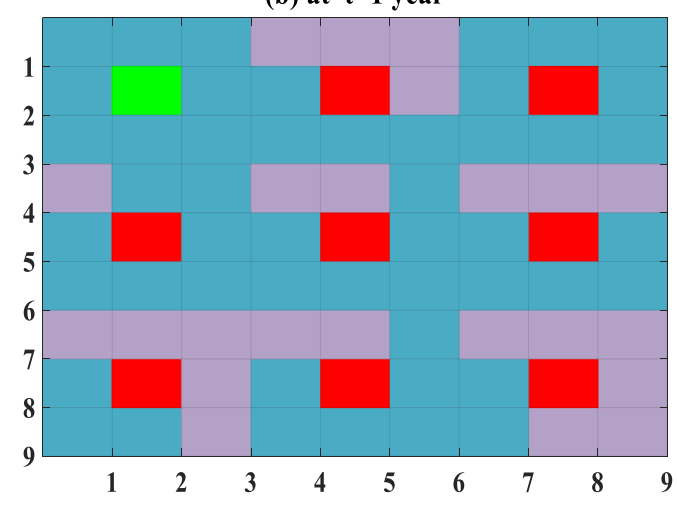


Fig. 4. Initial states of neurons and their synapses in CA model of sample $3 \times 3$ network at: (a) $t=0$, (b) $t=$

(a) at $\mathbf{t}=\mathbf{2}$ year (without any drugs)

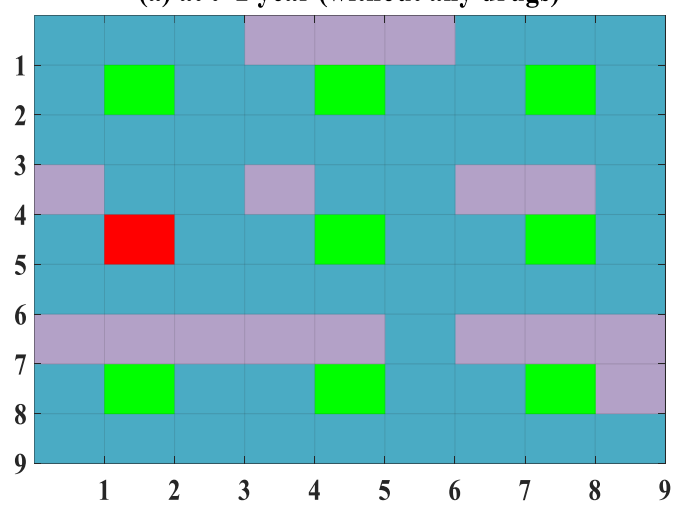

1 year

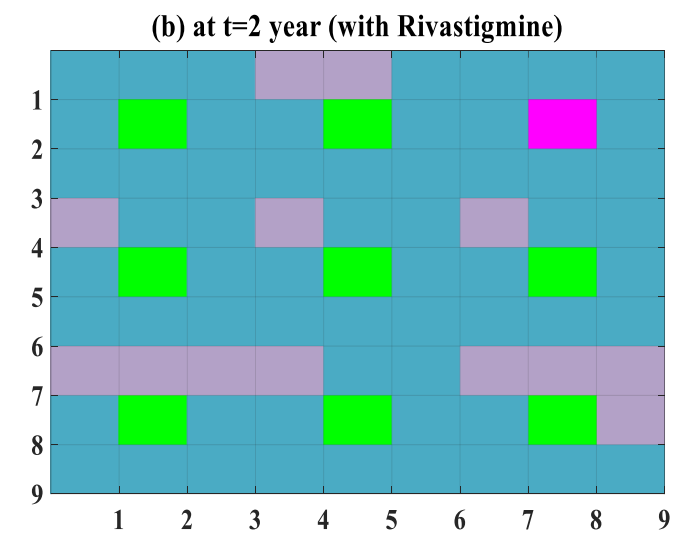

Fig. 5. CA model of neurons and their synapses in sample $3 \times 3$ network at $t=2$ years: (a) without any drugs, (b) with Rivastigmine.

(a) at $\mathbf{t}=\mathbf{2}$ year (without any drugs)

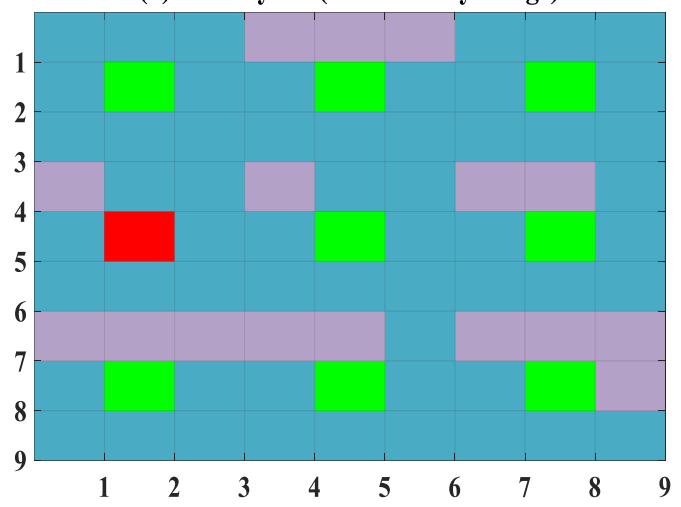

(b) at $\mathrm{t}=\mathbf{2}$ year (with Galantamine)

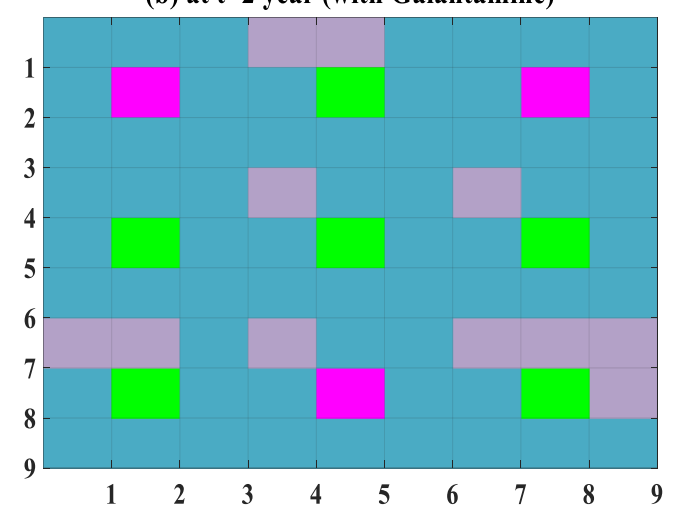

Fig. 6. CA model of neurons and their synapses in sample $3 \times 3$ network at $t=2$ years: (a) without any drugs, (b) with Galantamine.

Fig. 5 shows the analytical results of $\mathrm{CA}$ in sample $3 \times 3$ neuron network after two years, without any drugs and with the Rivastigmine injection. One can observe from Fig. 5 (a) that internal mechanisms decrease $\mathrm{CR}(C R \approx 11.1 \%)$ in the second year but the WR has reached $W R \approx 88.8 \%$ meaning that the system is already is in an emergency state. As shown in Fig. 5 (b), by injecting Rivastigmine during the second year, CR becomes zero and also the numbers of blocked synapses reduce. As a result, the Rivastigmine injection converts the critical state into a warning state, which is desirable.

To decrease the WR factor, a strong drug such as Galantamine can also be utilized. In Fig. 6, the analytical results of $\mathrm{CA}$ in a $3 \times 3$ neuron network after two years, with and without the Galantamine injection, are depicted. One can see from Fig. 6 (b) that $C R=0$ is similar to Rivastigmine injection. However, Galantamine has reduced the WR factor ( $W R \approx 66.6 \%$ ), which is better than Rivastigmine injection. 
As a final point, we studied the effect of Donepezil on the analytical results of $\mathrm{CA}$ in a $3 \times 3$ neuron system after two years, as shown in Fig. 7. It is clear from this figure that the CR factor reduces to zero by Donepezil Injection. Compared to other drugs i.e., Rivastigmine and Galantamine, the WR factor reaches $W R \approx 55.5 \%$, which is a great enhancement. Hence, utilizing a drug such as Donepezil can convert the emergency state into a warning state, and also give a lower WR factor in which the neurons' states are very stable in this situation.

The diagrams in Figures (8) to (10) show the effect of drugs on the critical and warning rate. The use of drugs improves the critical rate and warning rate in Alzheimer's disease.

(a) at $\mathrm{t}=\mathbf{2}$ year (without any drugs)

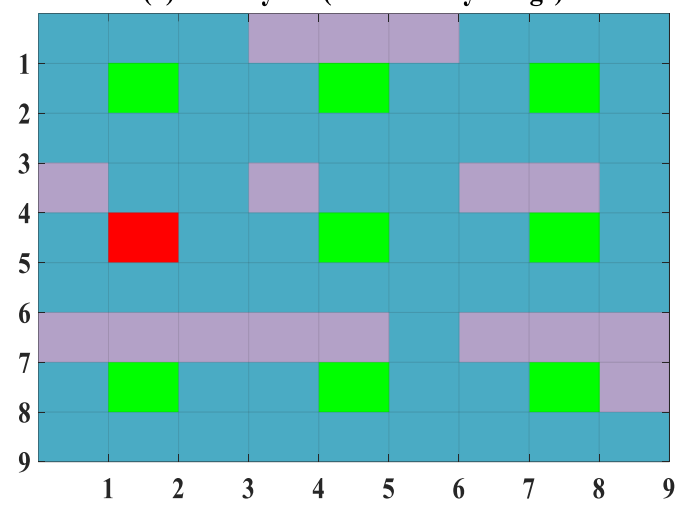

(b) at $\mathrm{t}=\mathbf{2}$ year (with Donepezil)

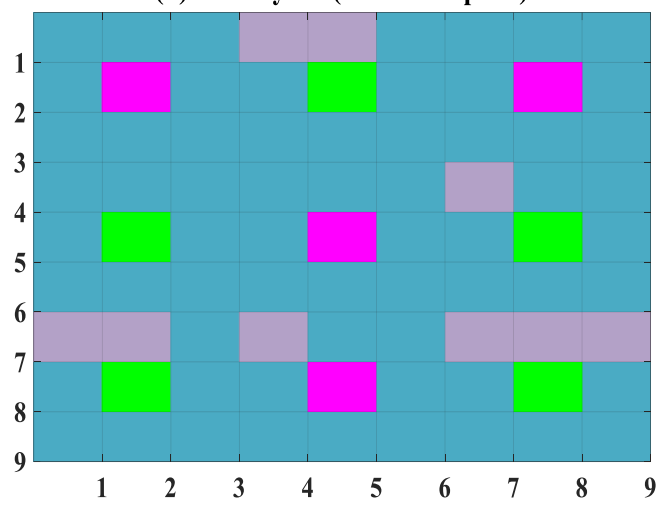

Fig. 7. CA model of neurons and their synapses in sample $3 \times 3$ network at $t=2$ years: (a) without any drugs, (b) with Donepezil.

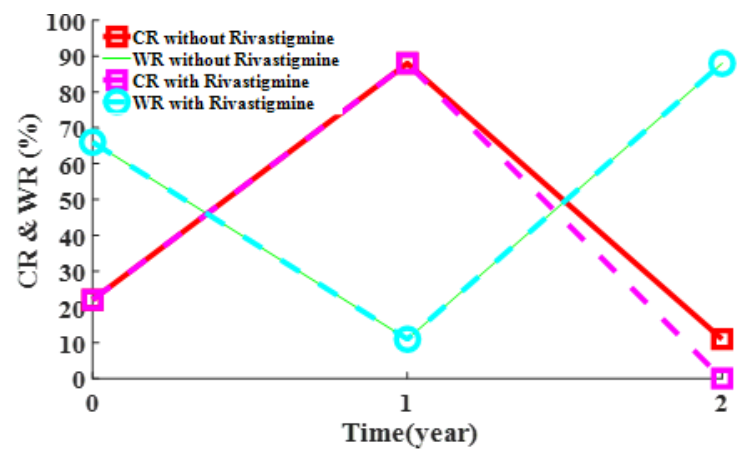

Fig. 8. The effect of Rivastigmine on critical and warning rate

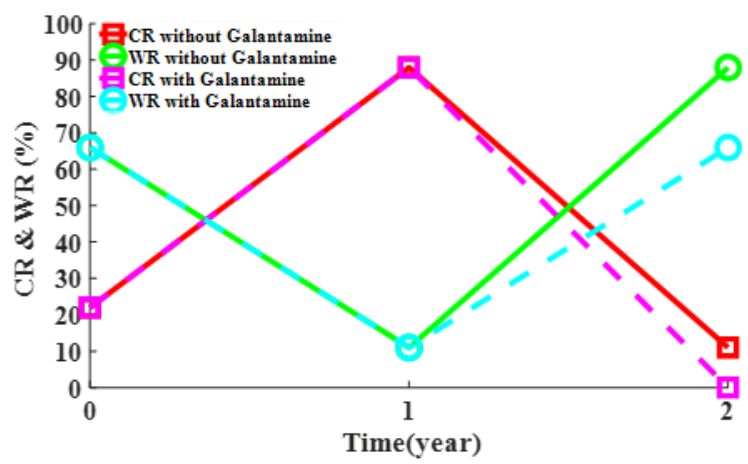

Fig .9. The effect of Galantamine on critical and warning rate 


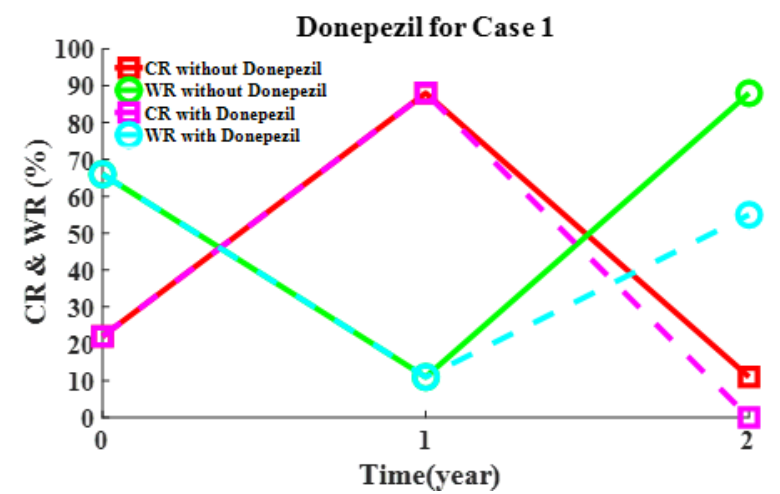

Fig .10. The effect of Donepezil on the critical and warning rate

\section{Conclusion}

In this paper, we proposed a new model for the study of drug injection on AD progress. This model was presented by the compounding of Puri-Li equations in the Cellular Automata. As a special case of general neuron network, a $3 \times 3$ neuron network was considered and the states of neurons and synapse cells were schematically shown. New definitions for AR, CR, and WR factors were presented to determine the stage of AD. It was shown that the drug injection can decrease the $\mathrm{AR}$ and CR factors to zero. Besides, choosing a strong drug such as Donepezil could reduce the WR factor, which is desirable. The proposed model can also be utilized for the study and forecasting the effect of various factors such as exercise, music, drugs, Gamma-ray, etc. on the AD progress.

\section{Declarations}

Ethical statement: This work is partially supported by Vice-Chancellor in Research Affair-Tabriz University of Medical Sciences under Ethical Code No. IR.TBZMED.VCR. REC.1399.377. The funders had no role in study design, analysis, numerical simulations, the decision to publish, or preparation of the manuscript.

Conflict of interest: The authors declare that they have no known competing financial interests or personal relationships that could have appeared to influence the work reported in this paper.

\section{References}

[1] V. Cutsuridis, Multiscale Models of Brain Disorders (no. 1). Springer, 2019.

[2] R. P. Mondaini, Trends in Biomathematics: Mathematical Modeling for Health, Harvesting, and Population Dynamics. Springer, 2019.

[3] J. Hardy and D. J. Selkoe, "The amyloid hypothesis of Alzheimer's disease: progress and problems on the road to therapeutics," science, vol. 297, no. 5580, pp. 353-356, 2002.

[4] I. K. Puri and L. Li, "Mathematical modeling for the pathogenesis of Alzheimer's disease," PloS one, vol. 5, no. 12, p. e15176, 2010. 
[5] W. Hao and A. Friedman, "Mathematical model on Alzheimer's disease," BMC systems biology, vol. 10, no. 1, pp. 1-18, 2016.

[6] R. Thuraisingham, "Examining the role of microglia and Astroglia during the pathogenesis of Alzheimer's disease via the Puri-li model," Journal of Molecular Neuroscience, vol. 62, no. 3, pp. 363-367, 2017.

[7] R. Thuraisingham, "Pathogenesis of Alzheimer's Disease Examined Using a Modified Puri-Li Model that Incorporates Calcium Ion Homeostasis," Journal of Molecular Neuroscience, vol. 65, no. 2, pp. 119-126, 2018.

[8] J. R. Petrella, W. Hao, A. Rao, and P. M. Doraiswamy, "Computational causal modeling of the dynamic biomarker cascade in Alzheimer's disease," Computational and mathematical methods in medicine, vol. 2019, 2019.

[9] C. R. Kyrtsos and J. S. Baras, "Modeling the role of the glymphatic pathway and cerebral blood vessel properties in Alzheimer's disease pathogenesis," PloS one, vol. 10, no. 10, p. e0139574, 2015.

[10] C. Hadjichrysanthou, A. K. Ower, F. de Wolf, R. M. Anderson, and A. s. D. N. Initiative, "The development of a stochastic mathematical model of Alzheimer's disease to help improve the design of clinical trials of potential treatments," PloS one, vol. 13, no. 1, p. $\mathrm{e} 0190615,2018$.

[11] M. A. Dayeh, G. Livadiotis, and S. Elaydi, "A discrete mathematical model for the aggregation of $\beta$-amyloid," PloS one, vol. 13, no. 5, p. e0196402, 2018.

[12] H.-Y. Jin and Z.-A. Wang, "A dual-gradient chemotaxis system modeling the spontaneous aggregation of microglia in Alzheimer's disease," Analysis and Applications, vol. 16, no. 03, pp. 307-338, 2018.

[13] J. De Caluwé and G. Dupont, "The progression towards Alzheimer's disease described as a bistable switch arising from the positive loop between amyloids and Ca2+," Journal of theoretical biology, vol. 331, pp. 12-18, 2013.

[14] M. Hoore, S. Khailaie, G. Montaseri, T. Mitra, and M. Meyer-Hermann, "Mathematical Model Shows How Sleep May Affect Amyloid- $\beta$ Fibrillization," Biophysical journal, vol. 119, no. 4, pp. 862-872, 2020.

[15] A. Lloret-Villas et al., "The impact of mathematical modeling in understanding the mechanisms underlying neurodegeneration: evolving dimensions and future directions," CPT: pharmacometrics \& systems pharmacology, vol. 6, no. 2, pp. 73-86, 2017.

[16] M. Bertsch, B. Franchi, N. Marcello, M. C. Tesi, and A. Tosin, "Alzheimer's disease: a mathematical model for onset and progression," Mathematical medicine and biology: a journal of the IMA, vol. 34, no. 2, pp. 193-214, 2017.

[17] B. Franchi, M. Heida, and S. Lorenzani, "A Mathematical model for Alzheimer's disease: An approach via stochastic homogenization of the Smoluchowski equation," arXiv preprint arXiv:1904.11015, 2019.

[18] C. R. Kyrtsos and J. S. Baras, "A graph theoretic mathematical model for Alzheimer's disease: Using a systems biology approach," in 2012 IEEE 12th International Conference on Bioinformatics \& Bioengineering (BIBE), 2012, pp. 472-477: IEEE.

[19] N. Kasabov, R. Schliebs, and H. Kojima, "Probabilistic computational neurogenetic modeling: From cognitive systems to Alzheimer's disease," IEEE Transactions on Autonomous Mental Development, vol. 3, no. 4, pp. 300-311, 2011. 